\title{
Updates on the relation of weight excess and reproductive function in men: sleep apnea as a new area of interest
}

\author{
Ahmad O Hammoud ${ }^{1}$, Douglas T Carrell ${ }^{1,2}$, Mark Gibson ${ }^{1}$, C Matthew Peterson $^{1}$ and A Wayne Meikle $^{3}$ \\ Obesity has a negative effect on male reproductive function. It is associated with low testosterone levels and alteration in gonadotropin \\ secretion. Male obesity has been linked to reduced male fertility. Data regarding the relation of obesity to sperm parameters are \\ conflicting in terms of the nature and magnitude of the effect. New areas of interest are emerging that can help explain the variation in \\ study results, such as genetic polymorphism and sleep apnea. Sleep disorders have been linked to altered testosterone production and \\ hypogonadism in men. It was also correlated to erectile dysfunction. The relation of sleep disorders to male fertility and sperm \\ parameters remains to be investigated. Men with hypogonadism and infertility should be screened for sleep apnea. Treatment of obesity \\ and sleep apnea improves testosterone levels and erectile function.
}

Asian Journal of Andrology (2012) 14, 77-81; doi:10.1038/aja.2011.64; published online 5 December 2011

Keywords: infertility; male infertility; reproductive function; sleep apnea; weight excess

\section{INTRODUCTION}

The effect of overweight and obesity on male reproductive function is gaining wide attention. Publications on this topic have moved from descriptive epidemiological manuscripts into mechanistic and interventional studies. ${ }^{1-3}$ This heightened interest is probably due to the fact that this association may be one of the unique causes of male infertility that may be potentially correctable using lifestyle modifications, or pharmacological and surgical interventions. While adequate randomized controlled studies of such interventions are lacking, preliminary reports appear promising. ${ }^{4}$ The effect of excess weight on male reproductive function is multifactorial. In this review, we will briefly present some of the known pathways, linking obesity-related metabolic changes to alterations in the hormonal profile and hence reproductive function in men. We will also explore some of the recent findings regarding sleep disorders, including sleep apnea, in relation to excess weight and reduced reproductive potential in men.

\section{DEFINITIONS}

Investigations on the effect of obesity on male reproductive potential are complicated by the use of different end points in various studies. Here, we propose a frame work that will aid in evaluating the literature related to this topic.

\section{Primary outcomes}

1. Male fertility: measured as fecundity (probability of pregnancy per menstrual cycle) or infertility (defined as no pregnancy after one year of unprotected intercourse), in the absence of female infertility factors.

2. Satisfaction with sexual life.

Intermediate outcomes

1. Alteration in spermatogenesis:

- sperm concentration;

- sperm motility;

- sperm morphology;

- sperm DNA fragmentation;

- surrogate markers of spermatogenesis, such as inhibin $\mathrm{B}$, anti-mullerian hormone $(\mathrm{AMH})$, etc.

2. Alteration of sexual performance (reduced coital frequency):

- erectile dysfunction;

- reduced libido.

3. Hormonal alteration:

- hypogonadism: reduced total and free testosterone;

- changes in sex hormone binding globulin;

- increased estradiol;

- altered gonadotropins secretion;

- effect of various adipose tissue hormones: leptin, ghrelin and adiponectin.

4. Genetic/epigenetic: affecting obesity and spermatogenesis, i.e., epigenetic modifications as an intermediate outcome with or without documented infertility.

${ }^{1}$ Division of Reproductive Endocrinology and Infertility, Department of Obstetrics and Gynecology, University of Utah School of Medicine, Salt Lake City, UT 84108, USA ${ }^{2}$ Andrology and IVF Laboratories, Department of Surgery (Urology) and Physiology, University of Utah School of Medicine, Salt Lake City, UT 84108, USA and ${ }^{3}$ Endocrinology/ Diabetes Research, Department of Medicine, University of Utah School of Medicine, Salt Lake City, UT 84132, USA

Correspondence: Dr AO Hammoud (ahmad.hammoud@hsc.utah.edu)

Received: 21 June 2011; Revised: 15 August 2011; Accepted: 24 August 2011; Published online: 5 December 2011 


\section{MALE OBESITY AND INFERTILITY}

Sallmen et $a l^{5}$ performed a secondary analysis of the Agricultural Health Study that included 1329 couples wherein data regarding body mass index (BMI) for both partners were available. Infertility was defined as an attempt at conception that lasted more than 12 months in the last 4 years. After correction for female BMI, male and female age, smoking status, alcohol use and exposure to solvents and pesticides, male BMI was shown to be weakly but statistically significantly associated with infertility with an odds ratio of 1.12 (95\% confidence interval: 1.01-1.25). There was a dose-effect maximal effect in the BMI class $32-43 \mathrm{~kg} \mathrm{~m}^{-2}$. $^{\circ}$

Ramlau-Hansen et al. ${ }^{6}$ analyzed data extracted from the Danish National Birth Cohort of 47835 women. Subfecundity was defined as an interval of more than 12 months to achieve a pregnancy that resulted in a live birth. After correction for female BMI and the age of both partners, overweight (BMI: $25-29.99 \mathrm{~kg} \mathrm{~m}^{-2}$ ) and obese (BMI: $\geqslant 30 \mathrm{~kg} \mathrm{~m}^{-2}$ ) males were more likely to have subfecundity with odds ratios of 1.15 (95\% confidence interval: $1.09-1.22$ ) and 1.49 (95\% confidence interval: 1.34-1.64). ${ }^{6}$ A secondary analysis of the Norwegian Mother and Child cohort study was performed by Nguyen et al. ${ }^{7}$ to evaluate the effect of male partner's weight on the couples' fertility. Infertility was defined as time to pregnancy of more than 12 months. After correcting for coital frequency, female BMI, male and female age, smoking status and various risk factors for female infertility, the odds ratio for infertility was 1.19 (95\% confidence interval: 1.03-1.62) for overweight men (BMI: 25-29.99 $\mathrm{kg} \mathrm{m}^{-2}$ ) and 1.36 (95\% confidence interval: $1.12-1.62)$ for obese men. ${ }^{7}$ In a much smaller study, Ohwaki and Yano ${ }^{8}$ showed increased incidence of not fathering a child per year of marriage with increasing BMI, in a group of 91 male Japanese auto workers. Interestingly, Jokela et al. ${ }^{9}$ studied the effect of increased weight in male adolescence on future fertility in 583 participants. The predicted number of future children was 1.46 for normal weight, 1.4 for overweight and 0.99 for the obese male adolescent. The association between adolescent's skinfold thickness and number of future children showed the same findings. Together, these studies suggest a small to moderate, dose-related effect of obesity on male fertility.

There are few studies exploring the effect of body weight in males and success of artificial insemination or in vitro fertilization. In a recent article, Bakos et al. ${ }^{2}$ recruited 305 couples undergoing initial in vitro fertilization treatment, to study the effect of paternal obesity on embryo quality and pregnancy rates. In this study, men with normal BMI had higher sperm concentration than overweight, obese and morbidly obese men. Morbidly obese men had a higher rate of requirement for intracytoplasmic sperm injection. After artificial reproductive technologies (ART), the rate of blastulation was higher in embryos created with sperm from normal weight men when compared to obese and severely obese men. There was a statistically significant linear reduction in pregnancy rates, clinical pregnancy rates and live birth rates with increasing BMI categories among the male partners. Live birth rates were the following: normal weight, $41.35 \%$; overweight, $26.4 \%$; obese, $22.6 \%$; and severely obese, $12.1 \%$. These striking results suggest abnormalities beyond the traditional sperm quality indices (count, motility and morphology), and may suggest alterations in the genetic or epigenetic programming of sperm from overweight and obese men.

\section{EFFECT OF MALE OBESITY ON INTERMEDIATE OUTCOMES OF MALE FERTILITY}

We and others have reviewed the effect of male overweight and obesity on numerous intermediate outcomes related to male fertility. ${ }^{10-12}$ An important potential marker is the alteration of semen parameters because of its obvious linkage to male fertility. Studies to date, however, are conflicted. A recent meta-analysis ${ }^{12}$ concluded that current evidence does not support a relation between increased body weight and reduced semen concentration. The main limitation of this meta-analysis was its restrictive nature; it included only six out of 13 identified studies. ${ }^{12}$ The six studies that were included had sperm concentration as the primary outcome. Studies that reported sperm morphology or composite outcomes such as total motile sperm count were excluded even though the outcomes, such as total motile sperm count, are more directly correlated to male fertility than sperm concentration. ${ }^{13-15} \mathrm{~A}$ more recent review of the topic concluded that obesity probably contributes to altered sperm concentration in a small group of obese men who likely have other risk factors that predispose to impaired spermatogenesis. ${ }^{16}$ To add more complexity to the matter, new evidence is emerging that the relation between male obesity and sperm parameters is modulated by genetic polymorphisms such as TTTA polymorphism in intron 4 of the aromatase gene through its alteration of estradiol levels. ${ }^{17,18}$

The relationship between male obesity and other intermediate outcomes such as lower testosterone levels (hypogonadism) and increased incidence of sexual dysfunction are less debated. ${ }^{3,12,16,19-21}$ The mechanisms accounting for reduced testosterone levels are various and include lowered sex hormone binding globulin (affecting primarily total testosterone) and the increased incidence of sleep apnea among overweight and obese men. ${ }^{10,22}$ Sleep apnea in this context is attracting attention because it can affect both testosterone levels (with potentially altered spermatogenesis) as well as, independently, erectile function. The combination of both factors may result in a compounding effect on male fertility.

\section{SLEEP, TESTOSTERONE AND MALE FERTILITY}

Several studies have shown that testosterone production is affected by the quality of sleep. There are two main states of sleep: rapid-eyemovement (REM) sleep and non-REM sleep. Non-REM sleep starts with a lighter sleep (stages 1 and 2), then progresses into deeper sleep (stages 3 and 4). REM sleep occurs at approximately 90-min intervals. These distinct states of sleep have characteristic patterns on both electroencephalography and electromyography. ${ }^{23}$ Sleep and sleep changes are thought to affect neuroendocrine function related to testosterone secretion. ${ }^{24,25}$ In the original study by Boyar et al., ${ }^{25}$ testosterone was found to increase in relation to an increase in luteinizing hormone (LH) production during sleep during early and mid-puberty. However, among a group of three adult men, only one man showed this characteristic pattern of increased testosterone during sleep. In a more recent study of seven healthy non-obese men, testosterone levels were compared during night sleep, night awake and day sleep. Maximum testosterone levels occurred during sleep for all individuals whether it was night sleep or day sleep. Testosterone levels appear to reach its maximal increase during the latter part of sleep and the first hour after awakening. ${ }^{24}$

Schiavi et al. ${ }^{26}$ recruited 67 healthy men (age: 45-75 years) to study pituitary gonadal function in relation to normal sleep and sleep disorders. Multiple hormones, including testosterone were sampled every $20 \mathrm{~min}$ during sleep. In this study, circulating testosterone, independent of age, was positively correlated to sleep efficiency, decreased latency to onset of REM activity and number of REM episodes. Bioavailable testosterone decreased with age, while sleep disordered breathing increased with age. The authors suggested that the age-related reduction in testosterone in this group of men is a result, 
in part, of the reduction in overnight testosterone caused by sleep disordered breathing. ${ }^{26}$ Later, Luboshitzky et al. ${ }^{27}$ explored whether the increase in testosterone levels during sleep are related to either REM sleep or represent a response to the phase of the circadian rhythm. Melatonin and core body temperature were used as markers of circadian rhythm. In this study, 10 young (ages: 22-26 years) healthy non-obese men (within $10 \%$ of ideal body weight) were subjected to a schedule of 7-min sleep and 13-min awake for $24 \mathrm{~h}$ (ultrashort sleep paradigm). In the same study, a group of six healthy men of similar age were followed during normal sleep from 12 midnight to 7 a.m. and were used as a control group. In both groups, blood samples were withdrawn every $20 \mathrm{~min}$. Sleep fragmentation by the 7/13 protocol blunted the rise in testosterone in most men. Men with fragmented sleep spent more time in stage one and less time in REM sleep when compared to controls. Despite its blunted rise, testosterone levels remained correlated to the onset of REM sleep. It is notable that none of five subjects with fragmented sleep, who did not have REM sleep, showed a sleep-related rise in testosterone. In this elegant study, the rise of testosterone was shown to coincide with the onset of REM sleep and was not correlated to the rise in melatonin (circadian rhythm). ${ }^{27}$

The exact mechanism causing the increased testosterone in relation to sleep stage is unknown; it is thought to be partially dependent on variation in LH levels. The sleep-related rise in testosterone is LHdependent in pubertal boys; however, only $15 \%$ of adult men show a nocturnal rise in LH levels leaving much of the nocturnal testosterone rise unexplained by gonadotropin changes with sleep. ${ }^{27}$ Walton et al. ${ }^{28}$ studied 24-h testosterone levels in men who were administered etonogestrel and testosterone to suppress the endogenous gonadotropins secretion compared to a control group. In the treated group, testosterone levels would be derived from the balance between the rate of release of testosterone from testosterone pellets and testosterone clearance. Epitestosterone was measured to reflect the remaining testicular steroidogenic function. Under this protocol, in the control group, testosterone levels did show a clear diurnal variation. This expected increase in testosterone at night with peak in the morning was, contrary to other reports, correlated to a rise in LH levels, suggesting that the diurnal variation in testosterone is due in part to a change in $\mathrm{LH}$ levels. In the treated group, epitestosterone also showed a diurnal variation with the highest elimination of epitestosterone in urine in the early morning, indicating another potential mechanism for the diurnal change in testicular function independent of the pituitary. ${ }^{28}$ In a later study, Luboshitzky et al. ${ }^{29}$ showed that men with obstructive sleep apnea had a difference in stage 2 and stages 3-4 sleep when compared to controls which correlated with lower LH and testosterone levels. This study suggested that the lower testosterone levels associated with sleep apnea may be attributed to differences in sleep patterns and sleep fragmentation. ${ }^{29}$ While breathing apparently affects testosterone levels, testosterone can also influence breathing. Testosterone supplementation in hypogonadal men is associated with increased prevalence of sleeping disorders. ${ }^{30,31}$ The exact role of testosterone in the control of the complex act of breathing remains undefined. ${ }^{32}$ Obstructive sleep apnea is more prevalent in males, consistent with the idea that testosterone may have a role in this disorder. ${ }^{31,33}$

\section{SLEEP DISORDERS, TESTOSTERONE AND MALE FERTILITY}

Studies exploring the effect of obesity on hormonal and semen parameters may be confounded by the effect of sleep disorders on these parameters. This effect of sleep disorders appears to be independent of BMI. Gambineri et al. ${ }^{34}$ showed that the reduction of testosterone levels in 15 patients with sleep apnea was independent of BMI or abdominal fatness. Luboshitzky et al. ${ }^{29}$ studied 10 patients with obstructive sleep apnea who were found to have a significantly lower mean and area-under-the-curve testosterone values when compared with controls. After correction for BMI, the difference in mean testosterone levels overall became non-significant, but the difference in area under the curve of testosterone through the night remained significant. In the same study, after correcting for BMI, area under the curve of LH (but not mean LH) was lower in men with obstructive sleep apnea when compared to controls. The authors concluded that central suppression is a possible cause for the lower testosterone levels associated with sleep apnea.

In a recently published study, our group reported on the effect of sleep apnea on reproductive hormones and its relation to BMI. Our patient population consisted of severely obese men (BMI: $\geqslant 35 \mathrm{~kg} \mathrm{~m}^{-2}$ ) recruited as part of the Utah Obesity Study. ${ }^{35}$ This study included 89 severely obese subjects who underwent various clinical measures including: a physician interview and detailed medical history, resting electroand echocardiograms, a submaximal exercise treadmill test and electrocardiogram, pulmonary function, polysomnography, resting metabolic rate, anthropometrics, resting and exercise blood pressure, comprehensive blood chemistry and urinalysis and dietary, quality of life and physical activity questionnaires.

The mean age of patients was $46.9 \pm 11.0$ years, the mean BMI was $47.8 \pm 8.7 \mathrm{~kg} \mathrm{~m}^{-2}$ and the mean weight was $337.7 \pm 62.4 \mathrm{lb}$. Sleep disorders were assessed using the following parameters. Hypopneas were defined as reduction of airflow for $\geqslant 10 \mathrm{~s}$ in conjunction with a $4 \%$ desaturation of pulse oximeter oxygen saturation $\left(\mathrm{SpO}_{2}\right) .{ }^{20} \mathrm{~A}$ respiratory disturbance index (RDI, events $\mathrm{h}^{-1}$ ) and a hypopnea index were calculated by dividing the total number of apneas/hypopneas by hours of recording. For the purpose of this study: no sleep apnea was defined as $\mathrm{RDI} \leqslant 5$ events $\mathrm{h}^{-1}$, mild sleep apnea $5<\mathrm{RDI} \leqslant 15$ events $\mathrm{h}^{-1}$, moderate sleep apnea $15<\mathrm{RDI} \leqslant 30$ events $\mathrm{h}^{-1}$ and severe apnea RDI $>30$ events $\mathrm{h}^{-1} .{ }^{21}$ Other parameters included mean $\mathrm{SpO}_{2}$, percent time below an $\mathrm{SpO}_{2}$ of $90 \%$ and percent time below an $\mathrm{SpO}_{2}$ of $80 \%$. The incidence of sleep apnea was $93.3 \%$. The distribution of the different degrees of sleep apnea was as follows: $6(6.7 \%)$ patients had no sleep apnea, $19(21.3 \%)$ had mild sleep apnea, $20(22.5 \%)$ had moderate sleep apnea and $44(49.4 \%)$ had severe sleep apnea. The adjusted means (corrected for age and BMI) of total testosterone per severity group of sleep apnea were as follows: no or mild sleep apnea: $386.51 \pm 27.34 \mathrm{ng} \mathrm{dl}^{-1}\left(13.41 \pm 0.95 \mathrm{nmol}^{-1}\right)$, moderate sleep apnea $331.90 \pm 30.11 \mathrm{ng} \mathrm{dl}^{-1}\left(11.51 \pm 1.04 \mathrm{nmol} \mathrm{l}^{-1}\right)$ and severe sleep apnea $275.00 \pm 20.50 \mathrm{ng} \mathrm{dl}^{-1}\left(9.54 \pm 0.71 \mathrm{nmol} \mathrm{l}^{-1}\right)(P=0.002)$. Free testosterone levels followed this pattern with statistical significance. Our analysis showed that an increase of 10 events in the RDI would result in $\sim 2 \mathrm{pg} \mathrm{ml}^{-1}\left(6.94 \mathrm{pmol}^{-1}\right)$ reduction in free testosterone levels. After correction for age and BMI, other parameters of sleep apnea including hypopnea index, percent time below an $\mathrm{SpO}_{2}$ of $90 \%$ and percent time below an $\mathrm{SpO}_{2}$ of $80 \%$ were also negatively correlated with total and free testosterone levels. ${ }^{22}$

There are few studies that explore the effect of continuous positive airway pressure (CPAP) therapy on the hormonal changes in men with sleep disordered breathing. Grunstein et al. ${ }^{36}$ reported in 1989 that sleep apnea (desaturation index: the hourly rate of episodes of arterial oxygen desaturation $>4 \%$ of the stable baseline) was independently associated with lower total and free testosterone levels. Parameters of sleep apnea at baseline were not associated with LH or follicle-stimulating hormone levels. In the same study, 43 men with severe sleep apnea were followed prospectively after 3 months of nasal continuous 
positive airway pressure (nCPAP). There was an increase in sex hormone binding globulin and total testosterone but not free testosterone levels. ${ }^{36}$ Luboshitzky et al. ${ }^{37}$ found similar changes in a smaller study that included five men who were followed for 9 months after CPAP. In contrast, Bratel et al. ${ }^{38}$ did not find a difference in testosterone levels after a follow-up period of 7 months of nCPAP in 11 men. However, at baseline, testosterone levels were normal despite the diagnosis of obstructive sleep apnea in all men. Testosterone levels in patients: $18.3 \pm 1.3 \mathrm{nmol}^{-1}$ were not different from those of controls $18.5 \pm 1.1 \mathrm{nmol}^{-1}$. The lack of improvement may be attributed to lack of effect of sleep apnea at baseline on testosterone levels in this group of patients. Interestingly, Santamaria et al. ${ }^{39}$ followed prospectively a group of 12 men who had lower testosterone levels in relation to obstructive sleep apnea and who had uvulopalatopharyngoplasty therapy. Three months after the surgery, there was improvement in testosterone levels $\left(13.31 \pm 1.07\right.$ to $\left.16.59 \pm 0.72 \mathrm{nmol} \mathrm{l}^{-1}, P<0.02\right)$, without significant changes in BMI, but with correlated improvement in breathing function. Meston et al. ${ }^{40}$ conducted a randomized trial where they assigned men with sleep apnea to either therapeutic nCPAP or placebo (the pressure was set to minimum with extra holes cut in the collar at the mask end of the connection tubing. This maintained pressure at a subtherapeutic level (about $1 \mathrm{~cm} \mathrm{H}_{2} \mathrm{O}$ ) and ensured no re-inhalation of $\mathrm{CO}_{2}$. The subjects were followed for 4 weeks, after that, the placebo group was crossed over to therapeutic nCPAP. There were a total of 101 patients that completed the trial, 52 had therapeutic nCPAP and 49 had placebo treatment. At baseline, hypopnea $(>4 \%$ $\mathrm{SpO}_{2}$ dips per hour) was correlated with lower $\mathrm{LH}$, follicle-stimulating hormone, total testosterone and sex hormone binding globulin (SHBG) levels. After 4 weeks of follow-up, in the treatment group there was no improvement in testosterone levels when compared to baseline $13.5 \pm 5.8 \mathrm{nmol} \mathrm{l}^{-1}$ vs. $13.2 \pm 4.7 \mathrm{nmol} \mathrm{l}^{-1}$. There was, however, a significant increase in SHBG levels. When compared to the placebo group, there was a difference in testosterone levels between groups; this was due largely to the reduction in testosterone level in placebo group. The lack of effect of nCPAP on testosterone levels could be attributed to the short period of treatment, 4 weeks as compared to the other studies. ${ }^{40}$

\section{SLEEP, SEXUAL FUNCTION AND MALE FERTILITY}

The association between sleep apnea and sexual dysfunction in men has also been explored independently or in association with changes in testosterone levels. This relationship has been suspected because of the association between testosterone and sleep-related erection. ${ }^{41}$ In fact, sleep-related erections are reduced in hypogonadal men and this pattern is reversed with testosterone supplementation. ${ }^{42}$ An altered sleep pattern that can affect testosterone levels might also be expected to contribute to erectile dysfunction by this mechanism. Semple et al. ${ }^{43}$ in 1984 reported on a man who presented with obesity, sleep apnea, whose reduced testosterone and impotence were reversed after weight loss. Schiavi et al. ${ }^{44}$ did not find any correlation between sleep disorders or respiratory flow studies and erectile dysfunction in older men. Margel et al. ${ }^{45}$ showed that only severe sleep apnea (RDI $>40)$ was associated with erectile dysfunction. In a more recent study, Stannek et al. ${ }^{46}$ concluded that age had a bigger influence on sexual functioning in men with sleep apnea than the severity of the disease. In another recent study that included 401 men who presented for sleep studies, sleep apnea measured as mean $\mathrm{SpO}_{2}$ was correlated with erectile dysfunction after correction for other risk factors for erectile dysfunction including age and BMI. However, a similar correlation was not found for other indices of sleep apnea (respiratory disturbance index, the lowest $\mathrm{SpO}_{2}$, desaturation index, arousal index) and erectile dysfunction. ${ }^{47}$ Andersen et al. ${ }^{48}$ studied the prevalence of erectile dysfunction in relation to sleep related breathing disorders in the population of Sao Paulo, Brazil. In this study a subset of 467 men representative of this population were included. All men answered questions related to sexual dysfunction and had polysomnography. Age more than 50 years, no physical activity, low testosterone levels, reduced time spent in REM and increased number of arousals were identified as risk factors for erectile dysfunction complains. In our study, severely obese men with sleep apnea had an overall reduced satisfaction with quality of sexual life. Increased severity of sleep apnea did not further reduce the scores of satisfaction with quality of sexual life, denoting a larger role for weight in this group of severely obese men with an average BMI of $47.8 \pm 8.7 \mathrm{~kg} \mathrm{~m}^{-2} .^{22}$ In conclusion, it appears that sleep apnea has an independent effect on erectile function; however, this effect is commonly eclipsed and confounded by the larger effect of obesity on both sleep apnea and erectile function.

\section{SLEEP AND MALE INFERTILITY}

To our knowledge, there are no studies exploring the fertility potential measured as fecundity rate or semen parameters in relation to sleep apnea. There are also no studies exploring the incidence of sleep apnea in the male partner in infertile couples or in males with unexplained abnormal semen parameters. In obese men, sleep apnea is likely to be an independent factor that contributes to the overall reduced fertile potential. Sleep apnea in obese men may independently exacerbate the negative effects on the reproductive system attributed to the various endocrine changes associated with obesity. Whether the testosterone change with sleep apnea and its improvement after CPAP therapy will be reflected in changes in the male's fertility, remains to be demonstrated. The effect of life style changes or pharmacological interventions targeted at correcting the negative effect of sleep apnea should be also a subject of interest for any future research.

\section{CONCLUSION}

The relationship between obesity and male hypogonadism is evident in numerous studies. There is also a clear negative relation between sexual function and increased weight in men. The relation between obesity and alteration in sperm parameters appears to be attenuated in comparison to the reduction in testosterone levels. However, the combination of hypogonadism and sexual dysfunction, and alteration in semen parameters result in reduced fertility at the population level. Sleep apnea, independently and in the context of obesity, is emerging as a risk factor for hypogonadism, sexual dysfunction and probably reduced fertility. Men with hypogonadism, erectile dysfunction and infertility should be screened for obesity and sleep apnea. Correction of these modifiable risk factors can improve the overall reproductive potential.

\section{COMPETING FINANCIAL INTERESTS}

The authors declare no competing financial interests.

1 Sallmén M, Sandler DP, Hoppin JA, Blair A, Baird DD. Reduced fertility among overweight and obese men. Epidemiology 2006; 17: 520-3.

2 Bakos HW, Henshaw RC, Mitchell M, Lane M. Paternal body mass index is associated with decreased blastocyst development and reduced live birth rates following assisted reproductive technology. Fertil Steril 2011; 95: 1700-4. 
3 Hammoud A, Gibson M, Hunt SC, Adams TD, Carrell DT et al. Effect of Roux-en-Y gastric bypass surgery on the sex steroids and quality of life in obese men. J Clin Endocrinol Metab 2009; 94: 1328-32.

4 Raman JD, Schlegel PN. Aromatase inhibitors for male infertility. J Urol 2002; 167: 624-9.

5 Sallmen M, Sandler DP, Hoppin JA, Blair A, Baird DD. Reduced fertility among overweight and obese men. Epidemiology 2006; 17: 520-3.

6 Ramlau-Hansen $\mathrm{CH}$, Thulstrup AM, Nohr EA, Bonde JP, Sorensen TI et al. Subfecundity in overweight and obese couples. Hum Reprod 2007; 22: 1634-7.

7 Nguyen R, Wilcox A, Skjaerven R, Baird DD. Men's body mass index and infertility. Hum Reprod 2007; 22: 2488-83.

8 Ohwaki K, Yano E. Body mass index as an indicator of metabolic disorders in annual health checkups among Japanese male workers. Ind Health 2009; 47: 611-6.

9 Jokela M, Kivimäki M, Elovainio M, Viikari J, Raitakari OT et al. Body mass index in adolescence and number of children in adulthood. Epidemiology 2007; 18: 599 606.

10 Hammoud AO, Gibson M, Peterson CM, Hamilton BD, Carrell DT. Obesity and male reproductive potential. J Androl 2006; 27: 619-26.

11 Hammoud AO, Gibson M, Peterson CM, Meikle AW, Carrell DT. Impact of male obesity on infertility: a critical review of the current literature. Fertil Steril 2008; 90: 897904.

12 MacDonald AA, Herbison GP, Showell M, Farquhar CM. The impact of body mass index on semen parameters and reproductive hormones in human males: a systematic review with meta-analysis. Hum Reprod Update 2010; 16: 293-311.

13 Ombelet W, Vandeput $\mathrm{H}$, van de Putte G, Cox A, Janssen M et al. Intrauterine insemination after ovarian stimulation with clomiphene citrate: predictive potential of inseminating motile count and sperm morphology. Hum Reprod 1997; 12: 145863

14 Miller DC, Hollenbeck BK, Smith GD, Randolph JF, Christman GM et al. Processed total motile sperm count correlates with pregnancy outcome after intrauterine insemination. Urology 2002; 60: 497-501.

15 Khalil MR, Rasmussen PE, Erb K, Laursen SB, Rex S et al. Homologous intrauterine insemination. An evaluation of prognostic factors based on a review of 2473 cycles. Acta Obstet Gynecol Scand 2001; 80: 74-81.

16 Teerds KJ, de Rooij DG, Keijer J. Functional relationship between obesity and male reproduction: from humans to animal models. Hum Reprod Update 2011; 17: 667 83.

17 Hammoud A, Carrell DT, Meikle AW, Xin Y, Hunt SC et al. An aromatase polymorphism modulates the relationship between weight and estradiol levels in obese men. Fertil Steril 2010; 94: 1734-8.

18 Hammoud AO, Griffin J, Meikle AW, Gibson M, Peterson CM et al. Association of aromatase (TTTAn) repeat polymorphism length and the relationship between obesity and decreased sperm concentration. Hum Reprod 2010; 25: 3146-51.

19 Giagulli VA, Kaufman JM, Vermeulen A. Pathogenesis of the decreased androgen levels in obese men. J Clin Endocrinol Metab 1994; 79: 997-1000.

20 Tchernof A, Despres JP, Belanger A, Dupont A, Prud'homme D et al. Reduced testosterone and adrenal C19 steroid levels in obese men. Metabolism 1995; 44 513-9.

21 Esposito K, Giugliano F, di Palo C, Giugliano G, Marfella R et al. Effect of lifestyle changes on erectile dysfunction in obese men: a randomized controlled trial. JAMA 2004: 291: 2978-84.

22 Hammoud AO, Walker JM, Gibson M, Cloward TV, Hunt SC et al. Sleep apnea reproductive hormones and quality of sexual life in severely obese men. Obesity (Silver Spring) 2011: 19: 1118-23

23 Andersen ML, Tufik S. The effects of testosterone on sleep and sleep-disordered breathing in men: its bidirectional interaction with erectile function. Sleep Med Rev 2008; 12: 365-79.

24 Axelsson J, Ingre M, Akerstedt T, Holmbäck U. Effects of acutely displaced sleep on testosterone. J Clin Endocrinol Metab 2005; 90: 4530-5.

25 Boyar RM, Rosenfeld RS, Kapen S, Finkelstein JW, Roffwarg HP et al. Human puberty. Simultaneous augmented secretion of luteinizing hormone and testosterone during sleep. J Clin Invest 1974; 54: 609-18.
26 Schiavi RC, White D, Mandeli J. Pituitary-gonadal function during sleep in healthy aging men. Psychoneuroendocrinology 1992; 17: 599-609.

27 Luboshitzky R, Zabari Z, Shen-Orr Z, Herer P, Lavie P. Disruption of the nocturna testosterone rhythm by sleep fragmentation in normal men. J Clin Endocrinol Metab 2001; 86: 1134-9.

28 Walton MJ, Anderson RA, Kicman AT, Elton RA, Ossowska K et al. A diurnal variation in testicular hormone production is maintained following gonadotrophin suppression in normal men. Clin Endocrinol (Oxf) 2007; 66: 123-9

29 Luboshitzky R, Lavie L, Shen-Orr Z, Herer P. Altered luteinizing hormone and testosterone secretion in middle-aged obese men with obstructive sleep apnea. Obes Res 2005; 13: 780-6.

30 Matsumoto AM, Sandblom RE, Schoene RB, Lee KA, Giblin EC et al. Testosterone replacement in hypogonadal men: effects on obstructive sleep apnoea, respiratory drives, and sleep. Clin Endocrinol (Oxf). 1985; 22: 713-21.

31 Sandblom RE, Matsumoto AM, Schoene RB, Lee KA, Giblin EC et al. Obstructive sleep apnea syndrome induced by testosterone administration. N Engl J Med 1983; 308: 508-10

32 Saaresranta T, Polo O. Hormones and breathing. Chest 2002; 122: 2165-82.

33 Block AJ, Boysen PG, Wynne JW, Hunt LA. Sleep apnea, hypopnea and oxygen desaturation in normal subjects. A strong male predominance. N Engl J Med 1979; 300: 513-7.

34 Gambineri A, Pelusi C, Pasquali R. Testosterone levels in obese male patients with obstructive sleep apnea syndrome: relation to oxygen desaturation, body weight, fat distribution and the metabolic parameters. J Endocrinol Invest 2003; 26: 493-8.

35 Adams TD, Avelar E, Cloward T, Crosby RD, Farney RJ et al. Design and rationale of the Utah obesity study. A study to assess morbidity following gastric bypass surgery. Contemp Clin Trials 2005; 26: 534-51.

36 Grunstein RR, Handelsman DJ, Lawrence SJ, Blackwell C, Caterson ID et al. Neuroendocrine dysfunction in sleep apnea: reversal by continuous positive airways pressure therapy. J Clin Endocrinol Metab 1989; 68: 352-8.

37 Luboshitzky R, Lavie L, Shen-Orr Z, Lavie P. Pituitary-gonadal function in men with obstructive sleep apnea. The effect of continuous positive airways pressure treatment. Neuro Endocrinol Lett 2003; 24: 463-7.

38 Bratel T, Wennlund A, Carlström K. Pituitary reactivity, androgens and catecholamines in obstructive sleep apnoea. Effects of continuous positive airway pressure treatment (CPAP). Respir Med 1999; 93: 1-7.

39 Santamaria JD, Prior JC, Fleetham JA. Reversible reproductive dysfunction in men with obstructive sleep apnoea. Clin Endocrinol (Oxf) 1988; 28: 461-70.

40 Meston N, Davies RJ, Mullins R, Jenkinson C, Wass JA et al. Endocrine effects of nasa continuous positive airway pressure in male patients with obstructive sleep apnoea. J Intern Med 2003; 254: 447-54.

41 Granata AR, Rochira V, Lerchl A, Marrama P, Carani C. Relationship between sleeprelated erections and testosterone levels in men. J Androl 1997; 18: 522-7.

42 Cunningham GR, Hirshkowitz M, Korenman SG, Karacan I. Testosterone replacement therapy and sleep-related erections in hypogonadal men. J Clin Endocrinol Metab 1990; 70: 792-7.

43 Semple PA, Graham A, Malcolm Y, Beastall GH, Watson WS. Hypoxia, depression of testosterone, and impotence in pickwickian syndrome reversed by weight reduction. Br Med J (Clin Res Ed) 1984; 289: 801-2.

44 Schiavi RC, Mandeli J, Schreiner-Engel P, Chambers A. Aging, sleep disorders, and male sexual function. Biol Psychiatry 1991; 30: 15-24.

45 Margel D, Cohen M, Livne PM, Pillar G. Severe, but not mild, obstructive sleep apnea syndrome is associated with erectile dysfunction. Urology 2004; 63: 545-9.

46 Stannek T, Hurny C, Schoch OD, Bucher T, Munzer T. Factors affecting self-reported sexuality in men with obstructive sleep apnea syndrome. J Sex Med 2009; 6: 341524

47 Budweiser S, Enderlein S, Jorres RA, Hitzl AP, Wieland WF et al. Sleep apnea is an independent correlate of erectile and sexual dysfunction. J Sex Med 2009; 6: 3147 57

48 Andersen ML, Santos-Silva R, Bittencourt LR, Tufik S. Prevalence of erectile dysfunction complaints associated with sleep disturbances in Sao Paulo, Brazil: a population-based survey. Sleep Med 2010; 11: 1019-24. 